\title{
The Asymptotic Behavior of Solutions of a Fractional Integro-differential Equation
}

\author{
AHMAD M. AHMAD \\ Prep Year Math Program \\ King Fahd University of Petroleum \& Minerals \\ Dhahran 31261 \\ SUADI ARABIA
}

\begin{abstract}
In this paper, we study the asymptotic behavior of solutions for an initial value problem with a nonlinear fractional integro-differential equation. Most of the existing results in the literature assume the continuity of the involved kernel. We consider here a kernel that is not necessarily continuous, namely, the kernel of the RiemannLiouville fractional integral operator that might be singular. We determine certain sufficient conditions under which the solutions, in an appropriate underlying space, behave eventually like power functions. For this purpose, we establish and generalize some well-known integral inequalities with some crucial estimates. Our findings are supported by examples and numerical calculations.
\end{abstract}

Key-Words: Fractional differential equation, Caputo fractional derivative, Riemann-Liouville fractional integral, initial value problem, asymptotic behavior, integral inequalities

Received: March 19, 2020. Revised: August 1, 2020. Accepted: August 20, 2020. Published: August 31, 2020.

\section{Introduction}

The fractional integro-differntial equation is a differential equation with arbitrary non-integer orders of derivatives and integrals. It is a generalization of the classical integro-differntial equation of integer order. During the last few decades, many investigations have shown that fractional integro-differntial equations can be mathematical models for several phenomena, processes and systems. Therefore, the theory of fractional differential equation attracted many researchers in engineering, physics, economy and other sciences.

In this article we are concerned with the asymptotic behavior of solutions for the following initial value problem

$$
\left\{\begin{array}{c}
\left({ }^{C} D_{0^{+}}^{\alpha} u\right)^{\prime}(t)=f\left(t, u(t),\left(I_{0^{+}}^{\beta} u\right)(t)\right), t \geq 0, \\
u(0)=u_{0},\left({ }^{C} D_{0^{+}}^{\alpha} u\right)(0)=u_{1}, u_{0}, u_{1} \in \mathbb{R} .
\end{array}\right.
$$

The derivative ${ }^{C} D_{0^{+}}^{\alpha}$ represent the Caputo fractional derivative of order $0<\alpha<1$. The operator $I_{0^{+}}^{\beta}$ is the Riemann-Liouville fractional integral of or$\operatorname{der} \beta>0$. The definitions of the Caputo fractional derivative and the Riemann-Liouville fractional integral are given in next section.

We use the fractional calculus theory and tools to treat the terms involving fractional derivatives and integrals. The associated Volterra integral equation corresponding to (1) is derived. We make use of some known or ad-hoc integral inequalities with some crucial estimates to the involved terms.
From both the theoretical and practical points of view, it is of great importance to have an idea about the behavior of solutions at large time-variable values. The importance of studying the asymptotic behavior of solutions of Problem (1) comes from the need to answer the following question "How do its solutions, which cannot be found explicitly behave as $t \rightarrow \infty$ ?".

It is known from the definition of fractional derivative that all the history of the state is taken into account through a convolution with (possibly) a singular kernel. Additionally, in our case, the nonlinear term involves an additional singularity, namely, the singular kernel of the Riemann-Liouville integral operator. Because of all these features, it is difficult to apply the existing approaches and methods in the literature for integer order to the noninteger order case.

The study of the linear asymptotic behavior of the solutions for linear and nonlinear differential equations is important in many fields like fluid mechanics, differential geometry, bidimensional gravity, Jacobi fields, etc. see, e.g., [16]. For instant, the existence of asymptotically linear solutions is related to many analytic properties like existence of nonoscillatory, monotonic, bounded, square integrable solutions and eventually positive (negative) solutions.

As a result, the topic of the asymptotic behavior of solutions for differential and integro-differential equations of integer and non-integer orders attracted many researchers e.g. see [8, 11, 13, 15, 17, 19, 20].

In [8], Băleanu et al. studied the asymptotic be- 
havior of solutions to the fractional differential equation

$$
D_{0^{+}}^{\alpha} x^{\prime}(t)+f(t, x)=0,0<\alpha<1, t>0,
$$

under the condition $|f(t, x)| \leq \phi\left(t, \frac{|x|}{(1+t)^{\alpha}}\right), t \geq 0$, $x \in \mathbb{R}$, where $D_{0^{+}}^{\alpha}$ is the Riemann-Liouville fractional derivative of order $\alpha, f:[0, \infty) \times \mathbb{R} \rightarrow \mathbb{R}$ is a continuous function and $\phi:[0, \infty) \times[0, \infty) \rightarrow$ $[0, \infty)$ is a continuous function and it is assumed to be nondecreasing in the second argument. They proved that the solution can be expressed asymptotically as $c_{1}+c_{2} t^{\alpha}+O\left(t^{\alpha-1}\right)$ when $t \rightarrow \infty, c_{1}, c_{2} \in \mathbb{R}$.

Medved showed in [19] that all solutions of the initial value problem

$$
\left\{\begin{array}{l}
{ }^{C} D_{a^{+}}^{\alpha+1} x(t)=f(t, x(t)), t \geq a>1,0<\alpha<1, \\
x(a)=c_{1}, x^{\prime}(a)=c_{2},
\end{array}\right.
$$

are asymptotic to a line for large values of $t$.

The authors of [17] considered the case when the source function $f$ depends on the solution and its $\mathrm{Ca}$ puto fractional derivative of a sub-order, namely

$$
\left\{\begin{array}{c}
{ }^{C} D_{a^{+}}^{\alpha} u(t)=f\left(t, u(t),{ }^{C} D_{a^{+}}^{\beta} u(t)\right), t \geq a, \\
u^{\prime}(a)=u_{0} .
\end{array}\right.
$$

where $a>0$ and $0<\beta<\alpha<1$. It has been shown that there exists a real number $b$ such that any global solution of Problem (2) is asymptotic to $b t^{\beta}$. For more kinds of asymptotic behaviors of solutions for other classes of fractional differential equations, the reader is advised to see the papers [4, 5, 9, 18, 19] and the references therein. Recently, the present author investigated the boundedness, power-type decay and asymptotic behavior of solutions for the following initial value problems with nonlinear fractional integro-differential equations

$$
\left\{\begin{array}{c}
\left({ }^{C} D_{0^{+}}^{\alpha} u\right)^{\prime}(t)=f\left(t, u(t), \int_{0}^{t} k(t, s, u(s)) d s\right), \\
u(0)=u_{0},{ }^{C} D_{0^{+}}^{\alpha} u\left(0^{+}\right)=u_{1}, u_{0}, u_{1} \in \mathbb{R},
\end{array}\right.
$$

and

$$
\begin{gathered}
\left\{\begin{array}{c}
{ }^{C} D_{0^{+}}^{\alpha} u=f\left(t,{ }^{C} D_{0^{+}}^{\beta} u, \int_{0}^{t} k\left(t, s,{ }^{C} D_{0^{+}}^{\gamma} u\right) d s\right), \\
I_{0^{+}}^{1-\alpha} u\left(0^{+}\right)=a_{1}, D_{0^{+}}^{\alpha} u\left(0^{+}\right)=a_{2}, a_{1}, a_{2} \in \mathbb{R},
\end{array}\right. \\
\text { where } t>0,0 \leq \beta \leq \alpha<1 \text { and } 0 \leq \gamma \leq \alpha<1 .
\end{gathered}
$$

Several different conditions are assumed on the source function $f$ and the kernel $k$ such as

$$
\begin{aligned}
|f(t, x, y)| & \leq h(t) p(|x|)+l(t) q(|y|), \\
|k(t, s, x)| & \leq w(s) r(|x|), \\
|k(t, s, x)| & \leq w(t, s) r(|x|), \text { or } \\
|k(t, s, x)| & \leq w(s) r\left(\frac{|x|}{t^{\alpha-\gamma-1}}\right)
\end{aligned}
$$

for some functions $h, l, p, q, r$ and $w$, see [4-6].

In all of the above papers, the continuity of the kernel $k$ on $[0, \infty)$ is assumed. In this paper we treat a kernel that is not necessarily continuous, namely, the kernel of the fractional integral operator $I_{0^{+}}^{\beta}$ that might be singular.

The well-posedness of solutions for a general class of fractional integro-differential equations of the type we deal with in this paper are established in [3].

In appropriate underlying spaces (such as the space of solutions we introduce in (6)), the continuity of the nonlinear term guarantees the local existence of solutions (see [1,-3, 7]). The local existence will be assumed throughout this paper. As our main concern is the asymptotic behavior of all possible solutions, we do not need the uniqueness of solutions.

The rest of this paper is organized as follows. In Section 2 we present some preliminary results. In particular, we list the basic definitions and properties of the involved fractional integrals and derivatives. Our main results are introduced in Section 3 As an application, we provide a supporting example with numerical calculations in Section 4. In the last section, we discuss and summarize our findings.

\section{Preliminaries}

In this section we introduce the definitions of the involved fractional derivative and integral and some of their basic properties. Bihari's Inequality and Pinto's lemma of nonlinear integral inequalities are presented at the end of this section.

Definition 1 The integral

$$
\left(I_{a+f}^{\alpha} f\right)(t)=\frac{1}{\Gamma(\alpha)} \int_{a}^{t}(t-\tau)^{\alpha-1} f(\tau) d \tau, a<t<b,
$$

is the Riemann-Liouville fractional integral of order $\alpha>0$, provided the right-hand side exists.

Definition 2 The derivative

$$
\left({ }^{C} D_{a^{+}}^{\alpha} f\right)(t)=\left(I_{a^{+}}^{1-\alpha} D f\right)(t) .
$$

is the Caputo fractional derivative of order $0 \leq \alpha<$ 1. In particular, ${ }^{C} D_{a^{+}}^{0} f=f,{ }^{C} D_{a^{+}}^{1} f=D f$.

The Riemann-Liouville fractional integral $I_{a+}^{\alpha}$ possesses the semigroup property as shown below.

Lemma 3 [14] Let $\alpha>0, \beta>0$, then

$$
I_{a+}^{\alpha} I_{a+}^{\beta} f=I_{a+}^{\alpha+\beta} f,
$$

almost everywhere in $[a, b]$ for $f \in L^{p}(a, b)$. When $f \in C[a, b]$, then this relation holds at every point in $[a, b]$. 
The Riemann-Liouville fractional integral of a power function yields a certain coefficient multiplied by that power function with the order of the fractional integral added to the power.

Lemma 4 [14] If $\alpha \geq 0, \beta>0$, then for $t>a$,

$$
\left(I_{a^{+}}^{\alpha}(\tau-a)^{\beta-1}\right)(t)=\frac{\Gamma(\beta)}{\Gamma(\beta+\alpha)}(t-a)^{\beta+\alpha-1} .
$$

The next lemma is about the composition of The Riemann-Liouville fractional integral and the Caputo fractional derivative.

Lemma 5 [14] Let $0<\alpha \leq 1$. If $f \in C^{1}[a, b]$, then

$$
\left(I_{a^{+}}^{\alpha}{ }^{C} D_{a^{+}}^{\alpha} f\right)(t)=f(t)-f(a), t \in(a, b] .
$$

In [4], We proved the following lemma that treats the limit of $\frac{1}{t^{\alpha}}\left(I_{a^{+}}^{\alpha+1} f\right)(t)$ as $t \rightarrow \infty$.

Lemma 6 [4] Let $f \in L^{1}\left(t_{0}, \infty\right), t_{0} \geq 0$ and $x$ and $y$ be real-valued functions defined on $\left[t_{0}, \infty\right)$, then

$$
\begin{aligned}
& \lim _{t \rightarrow \infty} \frac{1}{t^{\alpha}} \int_{t_{0}}^{t}(t-\tau)^{\alpha} f(\tau, x(\tau), y(\tau)) d \tau \\
&=\int_{t_{0}}^{\infty} f(\tau, x(\tau), y(\tau)) d \tau .
\end{aligned}
$$

Lemma 7 [10] (Bihari's Inequality) Let $y$ and $h$ be nonnegative continuous functions on $[a, \infty)$ and $g$ be positive on $(0, \infty)$ and continuous nondecreasing function on $[0, \infty)$. If

$$
y(t) \leq c+\int_{a}^{t} h(\tau) g(y(\tau)) d \tau, t \in[a, \infty),
$$

where the constant $c$ is positive, then

$$
y(t) \leq G^{-1}\left[G(c)+\int_{a}^{t} h(\tau) d \tau\right], t \in\left[a, b_{1}\right],
$$

where

$$
G(v)=\int_{v_{0}}^{v} \frac{d s}{g(s)}, v>0, v_{0}>0,
$$

$G^{-1}$ is the inverse function of $G$ and $b_{1}$ is chosen so that

$$
G(c)+\int_{a}^{t} h(\tau) d \tau \in \operatorname{Domain}\left(G^{-1}\right),
$$

for all $t \in\left[a, b_{1}\right]$.

As an extension for Bihari's inequality to a finite number of nonlinearities, Pinto proved, in [21], the following lemma.
Lemma 8 [21] Let $y, h_{i}, i=1, \ldots, n$ be nonnegative and continuous functions on $[a, b]$. Let $g_{i}, i=1, \ldots, n$ be nonnegative continuous and nondecreasing functions on $[0, \infty)$ such that $g_{1} \propto g_{2} \propto \ldots \propto g_{n}$ $\left(g_{n} / g_{n-1}, \ldots, g_{2} / g_{1}\right.$ are nondecreasing). Assume that $c$ is a a positive real number. If

$$
y(t) \leq c+\sum_{i=1}^{n} \int_{a}^{t} h_{i}(\tau) g_{i}(y(\tau)) d \tau, t \in[a, b],
$$

then, for $t \in\left[a, b_{1}\right]$,

$$
y(t) \leq G_{n}^{-1}\left(G_{n}\left(y_{n-1}\right)+\int_{a}^{t} h_{n}(\tau) d \tau\right),
$$

where

1. $G_{i}(v)=\int_{v_{i}}^{v} \frac{d s}{g_{i}(s)}, v>0, v_{i}>0, i=1, \ldots, n$ and $G_{i}^{-1}$ is the inverse function of $G_{i}$.

2. The constants $c_{i}$ are given by $y_{0}=c$ and $y_{i}=$ $G_{i}^{-1}\left(G_{i}\left(y_{i-1}\right)+\int_{a}^{b_{1}} h_{i}(\tau) d \tau\right), i=1, \ldots, n-$ 1.

3. The number $b_{1} \in[a, b]$ is the largest number such that

$$
\int_{a}^{b_{1}} h_{i}(\tau) d \tau \leq \int_{y_{i-1}}^{\infty} \frac{d s}{g_{i}(s)}, i=1, \ldots, n .
$$

\section{Main Results}

This section is devoted to the study of the asymptotic behavior of solutions for Problem (1).

By a solution $u$ for Problem (1), we mean a function $u:[0, \infty) \rightarrow \mathbb{R}$, that satisfies the equation and the initial conditions in (1) and is in the space $C^{\alpha, 1}[0, \infty)$ defined by

$$
C^{\alpha, 1}[0, \infty)=\left\{u \in C[0, \infty),\left({ }^{C} D_{0^{+}}^{\alpha} u\right)^{\prime} \in C[0, \infty)\right\} .
$$

The following result can be considered as a fractional version of L'Hospital's rule specified for the solution of Problem (1).

Lemma 9 Let u be a solution of Problem (1) with $f \in$ $L^{1}(0, \infty)$. Then,

$$
\begin{aligned}
& \lim _{t \rightarrow \infty} \frac{u(t)}{t^{\alpha}}=\lim _{t \rightarrow \infty} \frac{\left({ }^{C} D_{0^{+}}^{\alpha} u\right)(t)}{\Gamma(\alpha+1)} \\
& =\frac{1}{\Gamma(\alpha+1)}\left(u_{1}+\int_{0}^{\infty} f\left(s, u, I_{0^{+}}^{\beta} u\right) d s\right),
\end{aligned}
$$

and

$$
\frac{|u(t)|}{t^{\alpha}} \leq c_{1}+c_{2} \int_{0}^{t}\left|f\left(s, u(s),\left(I_{0^{+}}^{\beta} u\right)(s)\right)\right| d s,
$$


for all $t \geq 1$, where

$$
c_{1}=\left|u_{0}\right|+\frac{\left|u_{1}\right|}{\Gamma(\alpha+1)}, \quad c_{2}=\frac{1}{\Gamma(\alpha+1)} .
$$

Proof. Integrating both sides of the equation in (1) over the interval $[0, t]$ gives

$$
\left({ }^{C} D_{0^{+}}^{\alpha} u\right)(t)=u_{1}+\int_{0}^{t} f\left(s, u(s),\left(I_{0^{+}}^{\beta} u\right)(s)\right) d s .
$$

Applying $I_{a^{+}}^{\alpha}$ to both sides of equation $(9)$ yields with help of Lemmas 3, 4 and 5 ,

$$
u(t)=u_{0}+\frac{u_{1} t^{\alpha}}{\Gamma(\alpha+1)}+\left(I_{0^{+}}^{\alpha+1} f\left(s, u, I_{0^{+}}^{\beta} u\right)\right)(t) .
$$

Now, we divide both sides of (10) by $t^{\alpha}$, to obtain for $t>0$,

$\frac{u(t)}{t^{\alpha}}=\frac{u_{0}}{t^{\alpha}}+\frac{u_{1}}{\Gamma(\alpha+1)}+\frac{\left(I_{0^{+}}^{\alpha+1} f\left(s, u, I_{0^{+}}^{\beta} u\right)\right)(t)}{t^{\alpha}}$.

The limit (7) follows from (9) and Lemma 6

It is clear that the relationship (10) implies the following estimate on $|u(t)|$ for all $t>0$,

$$
\begin{aligned}
|u(t)| \leq & \left|u_{0}\right|+\frac{1}{\Gamma(\alpha+1)}\left(\left|u_{1}\right| t^{\alpha}\right. \\
& \left.+\int_{0}^{t}(t-s)^{\alpha}\left|f\left(s, u, I_{0^{+}}^{\beta} u\right)\right| d s\right) \\
\leq & \left|u_{0}\right|+\frac{\left|u_{1}\right| t^{\alpha}}{\Gamma(\alpha+1)} \\
& +\frac{t^{\alpha}}{\Gamma(\alpha+1)} \int_{0}^{t}\left|f\left(s, u, I_{0^{+}}^{\beta} u\right)\right| d s .
\end{aligned}
$$

Dividing by $t^{\alpha}, t \geq 1$, gives the inequality (8) and completes the proof.

Before presenting our results we start with the following two hypotheses on the nonlinear function $f$.

(A) $f(t, x, y)$ is a continuous function in $E=$ $\{(t, x, y): t \geq 0, x, y \in \mathbb{R}\}$ and there are functions $h:[0, \infty) \rightarrow[0, \infty)$ with $t^{2 \alpha+\beta} h(t) \in$ $L^{1}(1, \infty)$, and $g_{i}, i=1,2$ continuous nondecreasing on $[0, \infty)$ and positive on $(0, \infty)$ with $g_{i}(y) \leq x g_{i}\left(\frac{y}{x}\right), x \geq 1, y>0$ and $\int_{t_{0}}^{t} \frac{d \tau}{\left(g_{1} g_{2}\right)(\tau)} \rightarrow \infty$ as $t \rightarrow \infty$ for any $t_{0}>0$ such that

$|f(t, x, y)| \leq h(t) g_{1}(|x|) g_{2}(|y|),(t, x, y) \in E$.

(A) $f(t, x, y)$ is a continuous function in $E=$ $\{(t, x, y): t \geq 0, x, y \in \mathbb{R}\}$ and there are functions $h_{1}, h_{2}:[0, \infty) \rightarrow[0, \infty)$ with $t^{\alpha} h_{1}(t) \in$
$L^{1}(1, \infty), t^{\alpha+\beta} h_{2}(t) \in L^{1}(1, \infty)$ and functions $g_{i}, i=1,2$ continuous nondecreasing on $[0, \infty)$ and positive on $(0, \infty)$ with $g_{i}(y) \leq x g_{i}\left(\frac{y}{x}\right)$, $x \geq 1, y>0, \int_{t_{0}}^{t} \frac{d \tau}{g_{i}(\tau)} \rightarrow \infty$ as $t \rightarrow \infty$ for any $t_{0}>0$ and $g_{1} \propto g_{2}$ such that

$$
|f(t, x, y)| \leq h_{1}(t) g_{1}(|x|)+h_{2}(t) g_{2}(|y|),
$$

where $(t, x, y) \in E$.

For examples about functions satisfying the above hypotheses, see Example 14.

Lemma 10 If $u$ is a solution for the problem (1) and the function $f$ satisfies $\left(\boldsymbol{A}_{1}\right)$, then

$$
\frac{|u(t)|}{t^{\alpha}} \leq y(t), \frac{\left|\left(I_{0^{+}}^{\beta} u\right)(t)\right|}{t^{\alpha+\beta}} \leq y(t),
$$

for all $t \geq 1$ where

$$
y(t)=c_{1}+c_{2} \int_{0}^{t} h(s) g_{1}(|u(s)|) g_{2}\left(\left|\left(I_{0^{+}}^{\beta} u\right)(s)\right|\right) d s,
$$

$$
\begin{aligned}
c_{1}= & \max \left\{\left|u_{0}\right|+\frac{\left|u_{1}\right|}{\Gamma(\alpha+1)}, \frac{\left|u_{0}\right|}{\Gamma(\beta+1)}+\right. \\
& \left.\frac{\left|u_{1}\right|}{\Gamma(\alpha+\beta+1)}\right\}, \\
c_{2}= & \left\{\frac{1}{\Gamma(\alpha+1)}, \frac{1}{\Gamma(\alpha+\beta+1)}\right\} .
\end{aligned}
$$

Proof. Firstly, we integrate both sides of the equation of (1) over $[0, t]$, to get

$$
\left({ }^{C} D_{0^{+}}^{\alpha} u\right)(t)=u_{1}+\int_{0}^{t} f\left(s, u(s),\left(I_{0^{+}}^{\beta} u\right)(s)\right) d s .
$$

Next, applying $I_{a^{+}}^{\alpha}$ to both sides of equation (13) gives for, $t \geq 0$,

$$
u(t)=u_{0}+\frac{u_{1} t^{\alpha}}{\Gamma(\alpha+1)}+\left(I_{0^{+}}^{\alpha+1} f\left(s, u, I_{0^{+}}^{\beta} u\right)\right)(t),
$$

and

$|u(t)| \leq\left|u_{0}\right|+\frac{\left|u_{1}\right| t^{\alpha}}{\Gamma(\alpha+1)}+\frac{t^{\alpha} \int_{0}^{t}\left|f\left(s, u, I_{0^{+}}^{\beta} u\right)\right| d s}{\Gamma(\alpha+1)}$.

For $t \geq 1,14$ implies that

$$
\frac{|u(t)|}{t^{\alpha}} \leq\left|u_{0}\right|+\frac{\left|u_{1}\right|}{\Gamma(\alpha+1)}+\frac{\int_{0}^{t}\left|f\left(s, u, I_{0^{+}}^{\beta} u\right)\right| d s}{\Gamma(\alpha+1)} .
$$


From condition $\left(\mathbf{A}_{1}\right)$, we have for all $t \geq 1$,

$$
\begin{aligned}
\frac{|u(t)|}{t^{\alpha}} \leq & \left|u_{0}\right|+\frac{\left|u_{1}\right|}{\Gamma(\alpha+1)}+\frac{1}{\Gamma(\alpha+1)} \\
& \int_{0}^{t} h(s) g_{1}(|u|) g_{2}\left(\left|I_{0^{+}}^{\beta} u\right|\right) d s .
\end{aligned}
$$

Also, for all $t \geq 0$,

$$
\begin{aligned}
\left(I_{0^{+}}^{\beta} u\right)(t)= & I_{0^{+}}^{\beta}\left(u_{0}+\frac{u_{1} s^{\alpha}}{\Gamma(\alpha+1)}+\right. \\
& \left.\left(I_{0^{+}}^{\alpha+1} f\left(\tau, u, I_{0^{+}}^{\beta} u\right)\right)(s)\right)(t) \\
= & \frac{u_{0} t^{\beta}}{\Gamma(\beta+1)}+\frac{u_{1} t^{\alpha+\beta}}{\Gamma(\alpha+\beta+1)}+ \\
& \left(I_{0^{+}}^{\alpha+\beta+1} f\left(\tau, u, I_{0^{+}}^{\beta} u\right)\right)(t),
\end{aligned}
$$$$
\left|\left(I_{0^{+}}^{\beta} u\right)(t)\right| \leq \frac{\left|u_{0}\right| t^{\beta}}{\Gamma(\beta+1)}+\frac{t^{\alpha+\beta}}{\Gamma(\alpha+\beta+1)}
$$$$
\left(\left|u_{1}\right|+\int_{0}^{t} f\left|\left(s, u, I_{0^{+}}^{\beta} u\right)\right| d s\right) .
$$

For $t \geq 1$, we deduce that

$$
\begin{aligned}
\frac{\left|\left(I_{0^{+}}^{\beta} u\right)(t)\right|}{t^{\alpha+\beta}} \leq & \frac{\left|u_{0}\right|}{\Gamma(\beta+1)}+\frac{\left|u_{1}\right|}{\Gamma(\alpha+\beta+1)}+ \\
& \frac{\int_{0}^{t} h(s) g_{1}(|u|) g_{2}\left(\left|I_{0^{+}}^{\beta} u\right|\right) d s}{\Gamma(\alpha+\beta+1)}
\end{aligned}
$$

for all $t \geq 1$. The result is obtained from 15 and (16).

The next theorem represents the main result of this section.

Theorem 11 Suppose that the function $f$ satisfies $\left(\boldsymbol{A}_{1}\right)$, then there exists a real number $k$ such that any solution of Problem (1) is asymptotic to $k t^{\alpha}$ as $t \rightarrow \infty$.

Proof. Differentiating the expression $y$, given by (12), we obtain

$y^{\prime}(t)=c_{2} h(t) g_{1}(|u(t)|) g_{2}\left(\left|\left(I_{0^{+}}^{\beta} u\right)(t)\right|\right), t \geq 0$.

We concluded from (11) and the monotonicity of $g_{1}$ and $g_{2}$, that

$$
y^{\prime}(t) \leq c_{2} t^{2 \alpha+\beta} h(t) g_{1}(y(t)) g_{2}(y(t)) .
$$

Integrating both sides of $(17)$ over $[1, t]$ leads to $y(t) \leq y(1)+c_{2} \int_{1}^{t} s^{2 \alpha+\beta} h(s) g_{1}(y(s)) g_{2}(y(s)) d s$.
The functions $h$ and $g=g_{1} g_{2}$ satisfy the hypotheses of Bihari's Inequality (Lemma 7), thus

$y(t) \leq G^{-1}\left(G(c)+c_{2} \int_{1}^{t} s^{2 \alpha+\beta} h(s) d s\right):=c_{3}<\infty$,

for all $t \geq 1$, where $G^{-1}$ is the inverse of $G(s)=$ $\int_{s_{0}}^{s} \frac{d \tau}{\left(g_{1} g_{2}\right)(\tau)} s>0, s_{0}>0, c=y(1)$ and $c_{3}>0$ is a constant.

Recalling $(12)$ and $(13)$, we deduce, for all $t \geq 0$,

$$
\frac{\left|\left({ }^{C} D_{0^{+}}^{\alpha} u\right)(t)\right|}{\Gamma(\alpha+1)} \leq y(t) .
$$

In view of the relation $(11)$, we get, for all $t \geq 1$,

$$
\frac{\left|\left({ }^{C} D_{0^{+}}^{\alpha} u\right)(t)\right|}{\Gamma(\alpha+1)}, \frac{|u(t)|}{t^{\alpha}}, \frac{\left|\left(I_{0^{+}}^{\beta} u\right)(t)\right|}{t^{\alpha+\beta}} \leq c_{3} .
$$

Now,

$$
\begin{aligned}
& \int_{0}^{t}\left|f\left(s, u(s),\left(I_{0^{+}}^{\beta} u\right)(s)\right)\right| d s \\
\leq & \int_{0}^{t} h(s) g_{1}(|u(s)|) g_{2}\left(\left|\left(I_{0^{+}}^{\beta} u\right)(s)\right|\right) d s \\
\leq & \int_{0}^{1} h(s) g_{1}(|u(s)|) g_{2}\left(\left|\left(I_{0^{+}}^{\beta} u\right)(s)\right|\right) d s \\
+ & \int_{1}^{t} s^{2 \alpha+\beta} h(s) g_{1}\left(\frac{|u(s)|}{s^{\alpha}}\right) g_{2}\left(\frac{\left|I_{0^{+}}^{\beta} u\right|}{s^{\alpha+\beta}}\right) d s .
\end{aligned}
$$

The integral

$$
\int_{0}^{1} h(s) g_{1}(|u(s)|) g_{2}\left(\left|\left(I_{0^{+}}^{\beta} u\right)(s)\right|\right) d s
$$

is finite by the continuity of the integrands over $[0,1]$. Also,

$$
\int_{1}^{t} s^{2 \alpha+\beta} h(s) g_{1}\left(\frac{|u(s)|}{s^{\alpha}}\right) g_{2}\left(\frac{\left|\left(I_{0^{+}}^{\beta} u\right)(s)\right|}{s^{\alpha+\beta}}\right) d s
$$

is uniformly bounded by 20 and the assumption $t^{2 \alpha+\beta} h(t) \in L^{1}(1, \infty)$.

Hence the integral

$$
\int_{0}^{t} f\left(s, u(s),\left(I_{0^{+}}^{\beta} u\right)(s)\right) d s
$$

is absolutely convergent and

$$
\lim _{t \rightarrow \infty} \int_{0}^{t} f\left(s, u(s),\left(I_{0^{+}}^{\beta} u\right)(s)\right) d s<\infty .
$$


Therefore, in view of Lemma 6 and Lemma 9 , there exists a real number $k$ such that

$\lim _{t \rightarrow \infty} \frac{\left({ }^{C} D_{0^{+}}^{\alpha} u\right)(t)}{\Gamma(\alpha+1)}=\frac{u_{1}+\int_{0}^{\infty} f\left(s, u, I_{0^{+}}^{\beta} u\right) d s}{\Gamma(\alpha+1)}=k$,

and consequently $\lim _{t \rightarrow \infty} \frac{u(t)}{t^{\alpha}}=k$, which the desired result.

The next theorem shows that the same conclusion can be obtained with the hypothesis $\left(\mathbf{A}_{2}\right)$.

Theorem 12 Suppose that the function $f$ satisfies $\left(\boldsymbol{A}_{2}\right)$, then there exists a real number $c$ such that any solution of Problem (1) is asymptotic to $c t^{\alpha}$ as $t \rightarrow$ $\infty$.

Proof. An analogous process to the proof of Lemma 10, we have

$$
\frac{|u(t)|}{t^{\alpha}}, \frac{\left|\left(I_{0^{+}}^{\beta} u\right)(t)\right|}{t^{\alpha+\beta}} \leq v(t),
$$

for all $t \geq 1$, where

$$
\begin{aligned}
v(t)= & c_{1}+c_{2} \int_{0}^{t} h_{1}(s) g_{1}(|u(s)|) d s \\
& +c_{2} \int_{0}^{t} h_{2}(s) g_{2}\left(\left|I_{0^{+}}^{\beta} u\right|\right) d s, t \geq 0,
\end{aligned}
$$

Also, we obtain from (13) and (22) that

$$
\frac{\left|\left({ }^{C} D_{0^{+}}^{\alpha} u\right)(t)\right|}{\Gamma(\alpha+1)} \leq v(t) \text { forall } t \geq 0 .
$$

From $(22)$ and (21), we deduce that

$v^{\prime}(t) \leq c_{2} t^{\alpha} h_{1}(t) g_{1}(v(t))+c_{2} t^{\alpha+\beta} h_{2}(t) g_{2}(v(t))$,

and consequently,

$$
\begin{aligned}
v(t) \leq & v(1)+c_{2} \int_{1}^{t} s^{\alpha} h_{1}(s) g_{1}(v(s)) d s \\
& +c_{2} \int_{1}^{t} s^{\alpha+\beta} h_{2}(s) g_{2}(v(s)) d s .
\end{aligned}
$$

As the functions $h_{1}, h_{2}, g_{1}$ and $g_{2}$ satisfy the hypotheses of Lemma 8, then (24) gives, for all $t \geq 1$,

$$
v(t) \leq G_{2}^{-1}\left(G_{2}\left(v_{1}\right)+\int_{1}^{t} s^{\alpha+\beta} h_{2}(s) d s\right):=c_{4},
$$

$\mathrm{v}_{1}=G_{1}^{-1}\left(G_{1}\left(v_{0}\right)+\int_{1}^{t} s^{\alpha} h_{1}(s) d s\right)$, where $G_{i}^{-1}$ is the inverse of $G_{i}(s)=\int_{s_{0}}^{s} \frac{d \tau}{g_{i}(\tau)} s>0, s_{0}>0, v_{0}=$ $v(1)$ and $0<c_{4}<\infty$ is a constant.
Now, the estimates (21) and 23) yields

$$
\frac{\left|\left({ }^{C} D_{0^{+}}^{\alpha} u\right)(t)\right|}{\Gamma(\alpha+1)}, \frac{|u(t)|}{t^{\alpha}}, \frac{\left|\left(I_{0^{+}}^{\beta} u\right)(t)\right|}{t^{\alpha+\beta}} \leq c_{4},
$$

for all $t \geq 1$ and therefore

$$
\begin{aligned}
& \int_{0}^{t}\left|f\left(s, u(s),\left(I_{0^{+}}^{\beta} u\right)(s)\right)\right| d s \\
& \leq \int_{0}^{t}\left[h_{1}(s) g_{1}(|u|)+h_{2}(s) g_{2}\left(\left|I_{0^{+}}^{\beta} u\right|\right)\right] d s \\
& \leq \int_{0}^{1}\left[h_{1}(s) g_{1}(|u|)+h_{2}(s) g_{2}\left(\left|I_{0^{+}}^{\beta} u\right|\right)\right] d s \\
& +\int_{1}^{t} s^{\alpha} h_{1}(s) g_{1}\left(\frac{|u|}{s^{\alpha}}\right) d s \\
& +\int_{1}^{t} s^{\alpha+\beta} h_{2}(s) g_{2}\left(\frac{\left|I_{0^{+}}^{\beta} u\right|}{s^{\alpha+\beta}}\right) d s
\end{aligned}
$$

is uniformly bounded. The rest of the proof is quite similar to that of Theorem 11

Remark 13 The ordering and the monotonicity conditions on the functions $g_{1}$ and $g_{2}$ can be omitted using the functions

$$
\begin{aligned}
& \psi_{1}(t):=\max _{s \in[0, t]}\left\{g_{1}(s)\right\} \text { and } \\
& \psi_{2}(t):=\max _{s \in[0, t]}\left\{\frac{g_{2}(s)}{\psi_{1}(s)}\right\} \psi_{1}(t) .
\end{aligned}
$$

Note that the two functions $\psi_{1}$ and $\psi_{2}$ are nondecreasing nonnegative on $[0, \infty), g_{i}(t) \leq \psi_{i}(t), i=1,2$ for all $t \in[0, \infty)$ and $\psi_{1} \propto \psi_{2}$.

\section{Applications}

Our results can be applied to a variety of functions that appear in the literature. The next example shows that the class of the function in the hypothesis $\left(\mathbf{A}_{2}\right)$ is not empty and all the conditions of Theorem 12 are fulfilled. This example is followed by some numerical illustration.

Example 14 Consider the following fractional integro-differential equation

$$
\begin{aligned}
\left({ }^{C} D_{0^{+}}^{\alpha} u\right)^{\prime}(t)= & t^{\mu_{1}} e^{-t}(|u(t)|)^{\lambda_{1}} \\
& +t^{\mu_{2}} e^{-t}\left(\left(I_{0^{+}}^{\beta}|u|\right)(t)\right)^{\lambda_{2}}
\end{aligned}
$$

where $t>0,0<\alpha<1, \beta>0,0 \leq \lambda_{1} \leq \lambda_{2} \leq 1$ and $\mu_{1}, \mu_{2}>0$.

If we take

$h_{i}(t)=t^{\mu_{i}} e^{-\rho_{i} t}, g_{i}(t)=t^{\lambda_{i}}, 0<\rho_{i} \leq 1, i=1,2$, 
All the conditions of Theorem 12 are fulfilled

$$
\begin{aligned}
& \int_{1}^{\infty} t^{\alpha} h_{1}(t) d t<\int_{0}^{\infty} t^{\alpha} h_{1}(t) d t \\
& =\int_{0}^{\infty} t^{\alpha+\mu_{1}} e^{-\rho_{1} t} d t=\frac{\Gamma\left(\alpha+\mu_{1}+1\right)}{\rho_{1}^{\alpha+\mu_{1}+1}}<\infty \\
& \int_{1}^{\infty} t^{\alpha+\beta} h_{2}(t) d t<\int_{0}^{\infty} t^{\alpha+\beta} h_{2}(t) d t \\
& =\int_{0}^{\infty} t^{\alpha+\beta+\mu_{2}} e^{-\rho_{2} t} d t=\frac{\Gamma\left(\alpha+\beta+\mu_{2}+1\right)}{\rho_{2}^{\alpha+\beta+\mu_{2}+1}} \\
& <\infty, \\
& \int_{t_{0}}^{\infty} \frac{d t}{g_{i}(t)}=\int_{t_{0}}^{\infty} \frac{d t}{t^{\lambda_{i}}}=\infty, \\
& g_{i}(v)=v^{\lambda_{i}} \leq u^{1-\lambda_{i}} v^{\lambda_{i}}=u g_{i}\left(\frac{v}{u}\right), u \geq 1, \\
& v, t_{0}>0 .
\end{aligned}
$$

We conclude that every solution of (27) with $u(0)=$ $u_{0}$ and $\left({ }^{C} D_{0^{+}}^{\alpha} u\right)\left(0^{+}\right)=u_{1}$, is asymptotic to $\rho t^{\alpha}$ when $t \rightarrow \infty, \rho \in \mathbb{R}$.

For treating the problem in Example 14 numerically, we use the iterative scheme

$$
\begin{aligned}
u_{n}(t)= & u_{0}+\frac{u_{1} t^{\alpha}}{\Gamma(\alpha+1)}+I_{0^{+}}^{\alpha+1}\left(s^{\mu_{1}} e^{-t}\right. \\
& \left.\left(\left|u_{n-1}\right|\right)^{\lambda_{1}}+s^{\mu_{2}} e^{-s}\left(I_{0^{+}}^{\beta}\left|u_{n-1}\right|\right)^{\lambda_{2}}\right),
\end{aligned}
$$

$n=1,2, \ldots$. The curve of the first iteration shows, for $\mu_{1}=1, \mu_{2}=2, \mu_{3}=3, \alpha=\frac{1}{2}, \beta=2, \lambda_{1}=0$, $\lambda_{2}=1$, and $u_{0}=u_{1}=1$, that the graph of $u_{1}$ (red curve) is asymptotic to the curve $\rho t^{\alpha}$ (black curve) where $\rho=\lim _{t \rightarrow \infty} \frac{\left({ }^{C} D_{0^{+}}^{\alpha} u_{1}\right)}{\Gamma(\alpha+1)}=\frac{28}{\sqrt{\pi}}$.

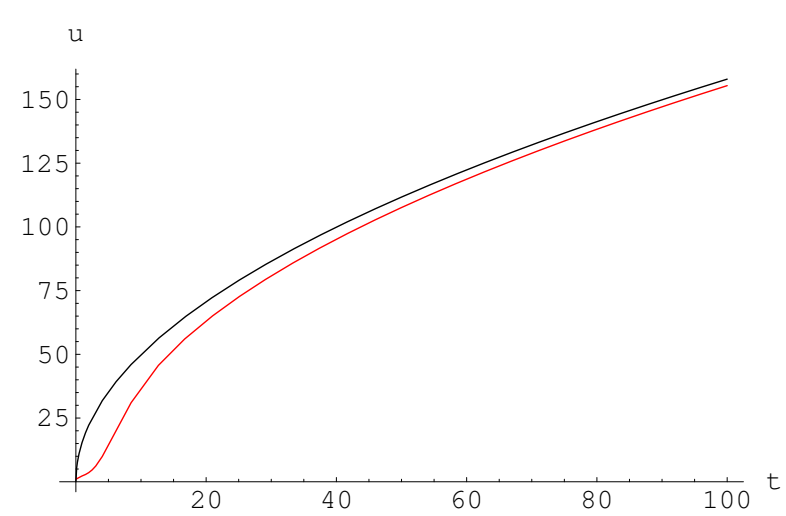

Figure 1: Figure 1: $\mu_{1}=1, \mu_{2}=2, \mu_{3}=3, \alpha=\frac{1}{2}$, $\beta=2, \lambda_{1}=0, \lambda_{2}=1$ and $u_{0}=u_{1}=1$

\section{Discussion and Conclusion}

In this paper, we considered an initial value problem with a fractional integro-differential equation involving the Caputo fractional derivative on the lefthand side. The nonlinear source function in the righthand side depends on the solution and the RiemannLiouville fractional integral operator of the solution. In general, the solution of Problem (1) cannot be found explicitly.

Unlike most of the existing results, we do not require the continuity of the involved kernel. We assumed the boundedness of the nonlinear term by sums or products of continuous functions of time, in certain Lebesgue spaces, and nondecreasing functions of the states. Under these conditions, we have found that the solutions of Problem (1) behave eventually like the solutions of the associated linear equation with a zero right-hand side.

Our results open many possible directions for future works. The fractional derivative we have considered is of Caputo type, $D\left({ }^{C} D_{0^{+}}^{\alpha}\right)$ where $D=\frac{d}{d t}$ and $0<\alpha<1$. Indeed, it would be interesting to consider higher order derivatives such as the fractional derivative ${ }^{C} D_{0^{+}}^{\alpha}, 1<\alpha<2$ without splitting it into two derivatives, one usual and one fractional.

Other types of fractional derivatives can be considered in future studies. The asymptotic behavior of solutions can be investigated under other kinds of boundedness and growth conditions on the nonlinearities.

\section{Acknowledgment}

The author would like to acknowledge the support provided by King Fahd University of Petroleum \& Minerals through Project SB191023.

\section{References:}

[1] R. P. Agarwal, M. Benchohra, and S. Hamani. A survey on existence results for boundary value problems of nonlinear fractional differential equations and inclusions. Acta Applicandae Mathematicae, 109(3):973-1033, 2010.

[2] R. P. Agarwal, S. K. Ntouyas, B. Ahmad, and M. S. Alhothuali. Existence of solutions for integro-differential equations of fractional order with nonlocal three-point fractional boundary conditions. Advances in Difference Equations, 2013(1):1-9, 2013.

[3] A. Aghajani, Y. Jalilian, and J. Trujillo. On the existence of solutions of fractional integrodifferential equations. Fractional Calculus and Applied Analysis, 15(1):44-69, 2012. 
[4] A. M. Ahmad, K. M. Furati, and N.-E. Tatar. Asymptotic power type behavior of solutions to a nonlinear fractional integro-differential equation. Electronic Journal of Differential Equations, 2017(134):1-16, 2017.

[5] A. M. Ahmad, K. M. Furati, and N.-E. Tatar. Asymptotic behavior of solutions for a class of fractional integro-differential equations. Mediterranean Journal of Mathematics, 15(5):188, 2018.

[6] A. M. Ahmad, K. M. Furati, and N.-E. Tatar. Boundedness and power-type decay of solutions for a class of generalized fractional langevin equations. Arabian Journal of Mathematics, 8(2):79-94, 2019.

[7] A. Anguraj, P. Karthikeyan, and J. Trujillo. Existence of solutions to fractional mixed integrodifferential equations with nonlocal initial condition. Advances in Difference Equations, 2011(1):690653, 2011.

[8] D. Băleanu, R. P. Agarwal, O. G. Mustafa, and M. Coşulschi. Asymptotic integration of some nonlinear differential equations with fractional time derivative. Journal of Physics A: Mathematical and Theoretical, 44(5):055203, 2011.

[9] D. Băleanu, O. G. Mustafa, and R. P. Agarwal. Asymptotic integration of $(1+\alpha)$-order fractional differential equations. Computers \& Mathematics with Applications, 62(3):1492-1500, 2011.

[10] I. Bihari. A generalization of a lemma of Bellman and its application to uniqueness problems of differential equations. Acta Mathematica Hungarica, 7(1):81-94, 1956.

[11] E. Brestovanska and M. Medved'. Asymptotic behavior of solutions to second-order differential equations with fractional derivative perturbations. Electronic Journal of Differential Equations, 2014(201):1-10, 2014.

[12] F. M. Dannan. Integral inequalities of GronwallBellman-Bihari type and asymptotic behavior of certain second order nonlinear differential equations. Journal of Mathematical Analysis and Applications, 108(1):151-164, 1985.

[13] S. R. Grace. On the oscillatory behavior of solutions of nonlinear fractional differential equations. Applied Mathematics and Computation, 266:259-266, 2015.

[14] A. A. Kilbas, H. M. Srivastava, and J. J. Trujillo. Theory and applications of fractional differential equations, volume 204. Elsevier Science Limited, 2006.

[15] T. Kusano and W. F. Trench. Existence of global solutions with prescribed asymptotic behavior for nonlinear ordinary differential equations. Annali di Matematica Pura ed Applicata, 142(1):381-392, 1985.

[16] S. Lang. Fundamentals of Differential Geometry. Volume 191 of Graduate Texts in Mathematics. Springer-Verlag, New York, 1999.

[17] M. Medved' and M. Pospíšil. Asymptotic integration of fractional differential equations with integrodifferential right-hand side. Mathematical Modelling and Analysis, 20(4):471-489, 2015.

[18] M. Medved. On the asymptotic behavior of solutions of nonlinear differential equations of integer and also of non-integer order. Electronic Journal of Qualitative Theory of Differential Equations, 10:1-9, 2012.

[19] M. Medved'. Asymptotic integration of some classes of fractional differential equations. Tatra Mountains Mathematical Publications, 54(1):119-132, 2013.

[20] O. G. Mustafa and D. Baleanu. On the asymptotic integration of a class of sublinear fractional differential equations. arXiv preprint arXiv:0904.1495, 2009.

[21] M. Pinto. Integral inequalities of Biharitype and applications. Funkcialaj Ekvacioj, 33(3):387-403, 1990.

\section{Creative Commons Attribution License 4.0 (Attribution 4.0 International, CC BY 4.0)}

This article is published under the terms of the Creative Commons Attribution License 4.0

https://creativecommons.org/licenses/by/4.0/deed.en_US 